\title{
Erratum to: Optimization of Surface Registrations Using Beltrami Holomorphic Flow
}

\author{
Lok Ming Lui · Tsz Wai Wong • Wei Zeng • \\ Xianfeng Gu • Paul M. Thompson - Tony F. Chan • \\ Shing-Tung Yau
}

Published online: 16 September 2011

(C) Springer Science+Business Media, LLC 2011

\section{Erratum to: J Sci Comput \\ DOI 10.1007/s10915-011-9506-2}

The affiliation of Prof. Shing Tung Yau was incorrectly listed as the Chinese University of Hong Kong. The correct affiliation should be: Department of Mathematics, Harvard University, Boston, MA, USA.

The online version of the original article can be found under doi:10.1007/s10915-011-9506-2.

L.M. Lui $(\varangle)$

Department of Mathematics, The Chinese University of Hong Kong, Shatin, New Territories, Hong Kong

e-mail: lmlui@math.cuhk.edu.hk

T.W. Wong

Department of Mathematics, University of California, Los Angeles, USA

W. Zeng $\cdot$ X. Gu

Department of Computer Science, State University of New York at Stony Brook, New York, USA

P.M. Thompson

Laboratory of Neuroimaging, UCLA Medical School, Los Angeles, USA

T.F. Chan

Hong Kong University of Science and Technology, Clear Water Bay, Kowloon, Hong Kong

S.-T. Yau

Department of Mathematics, Harvard University, Boston, MA, USA 